\title{
Flow-Based Assembly of Layer-by-Layer Capsules through Tangential Flow Filtration
}

Mattias Björnmalm, Ali Roozmand, Ka Fung Noi, Junling Guo, Jiwei Cui, Joseph J. Richardson ${ }^{\dagger}$, and Frank Caruso*

ARC Centre of Excellence in Convergent Bio-Nano Science and Technology, and the Department of Chemical and Biomolecular Engineering, The University of Melbourne, Parkville, Victoria 3010, Australia.

Keywords: cross-flow filtration, nanoengineering, automated layer-by-layer, multilayered particles 


\begin{abstract}
Layer-by-layer (LbL) assembly on nano- and microparticles is of interest for a range of applications, including catalysis, optics, sensors and drug delivery. One current limitation is the standard use of manual, centrifugation-based (pellet/resuspension) methods to perform the layering steps, which can make scalable, highly controllable and automatable production difficult to achieve. Here, we develop a fully flow-based technique using tangential flow filtration (TFF) for LbL assembly on particles. We demonstrate that multilayered particles and capsules with different sizes (from micrometers to sub-micrometers in diameter) can be assembled on different templates (e.g., silica and calcium carbonate) using several polymers (e.g., poly(allylamine hydrochloride), poly(styrene sulfonate) and poly(diallyldimethylammonium chloride)). The full system only contains fluidic components routinely used (and automated) in industry, such as pumps, tanks, valves and tubing in addition to the TFF filter modules. Using the TFF LbL system, we also demonstrate the centrifugation-free assembly, including core dissolution, of drug-loaded capsules. The well-controlled, integrated and automatable nature of the TFF LbL system provide scientific, engineering and practical processing benefits, making it valuable for research environments, and potentially useful for translating LbL assembled particles into diverse applications.
\end{abstract}




\section{INTRODUCTION}

Layer-by-layer (LbL) assembled materials and coatings are of interest because of their potential in applications ranging from optics and catalysis to energy and biomedicine. ${ }^{1-8}$ The properties of the assembled materials can be controlled through selection of the template, coating material, assembly conditions as well as the assembly technology used. ${ }^{9-11}$ Using particles as templates, layer-by-layer (LbL) assembly can also be used to engineer core-shell particles and capsules. ${ }^{12,13}$

Multilayered particles and capsules can be produced through several different methods and techniques, each offering distinct material and processing advantages. ${ }^{9}$ Sequential immersion of particulate templates into complementary layering solutions (e.g., oppositely charged polyelectrolytes) with intermediate centrifugation-based purification steps is the most wellstudied assembly method and is one of the most commonly used. ${ }^{12,13}$ Other examples include centrifugation-free calculated-saturation, ${ }^{14}$ immobilization-based techniques, ${ }^{15-18}$ atomization, ${ }^{19}$ magnetic separation, ${ }^{20}$ creaming, ${ }^{21}$ filtration, ${ }^{22-24}$ and macro- and microfluidic approaches. ${ }^{25-29}$ Recently, there has also been increasing interest in “single-step” preparation methods of polymer microcapsules, for example, using interfacial complexation or cross-linking, ${ }^{30,31}$ or using ultrasonic spraying and salt diffusion. ${ }^{32}$ Although all of these techniques have specific strengths and enable particle systems to be engineered using LbL assembly, some have restrictions on the diversity of the particles and materials that can be employed, while others require extensive manual intervention and hands-on time during the assembly process, thus making automation, scale-up and robust and reproducible production challenging to achieve. ${ }^{9}$

Precise and highly controlled processes, with minimal to no manual intervention, can be engineered using flow-based automated closed-loop systems. Automated systems for the production of peptides $^{33}$ and oligonucleotides ${ }^{34}$ have revolutionized the fields of biotechnology 
and biomedicine, and recent developments in automated synthesis of organic small molecules have the potential to have a similar impact. ${ }^{35,36}$ Flow-based closed-loop systems are also commonly used in industry, as they can ensure well-controlled, reliable and cost-effective production. One method of creating a flow-based systems is using filter membranes. Voigt et al. have previously demonstrated that a dead-end filtration system can be used for layer-by-layer assembly, ${ }^{22}$ but dead-end filtration is not suitable for continuous flow-based closed-loop systems (as the retentate is continuously forced against the filter and is not mobile) and filter caking can be an issue. Therefore, in high-throughput separation processes of high-value products - such as protein separation in the biopharmaceutical industry - tangential flow filtration (TFF; also known as cross-flow filtration) is commonly used instead of dead-end filtration. ${ }^{37-39}$

Herein, we develop a fluidic set-up for LbL assembly on micrometer and sub-micrometer particulate templates using hollow fiber TFF (Figure 1). In this approach, polymers are mixed with particulate templates, incubated to allow layering, and then excess polymer is removed using TFF. The next layer material can then be added to the purified particles which, again after incubation, can be purified through TFF. This process can then be repeated until the desired numbers of layers are deposited on the particles. The end products are multilayer core-shell particles or capsules following core-removal.

Besides the TFF filter modules, the main components of the flow-based TFF system for LbL assembly are pumps, tanks, valves, and tubing, which are all standard commercially available and industrially used fluidic components. All of these components are amenable to fluidic integration, enabling the design of a fully automated system, which can provide scientific, engineering and practical processing benefits. When the particles remain in a closed-loop system the assembly conditions can be highly controlled. As only pumps and switching of valves are 
used to control both the layering and washing conditions (time, volume, turbulence etc.), this can be used to optimize the assembly and washing conditions, which can have a direct impact on the resulting nanofilm properties and performance. ${ }^{9}$ Furthermore, automated systems have direct practical benefits, including minimal to no manual intervention required, increased robustness and reproducibility, and scalability and high-throughput. These are all critical for any system that aims to be industrially relevant. In addition, closed systems can enable contamination-free (or even sterile) assembly conditions, crucial for many biomedical applications, as well as the recovery and reuse of valuable materials, such as custom-designed polymers or therapeutics. LbL assembly through TFF thereby addresses key challenges associated with the assembly of multilayered particles and capsules, through a set-up that only uses standard fluidic equipment together with commercially available TFF filter modules.

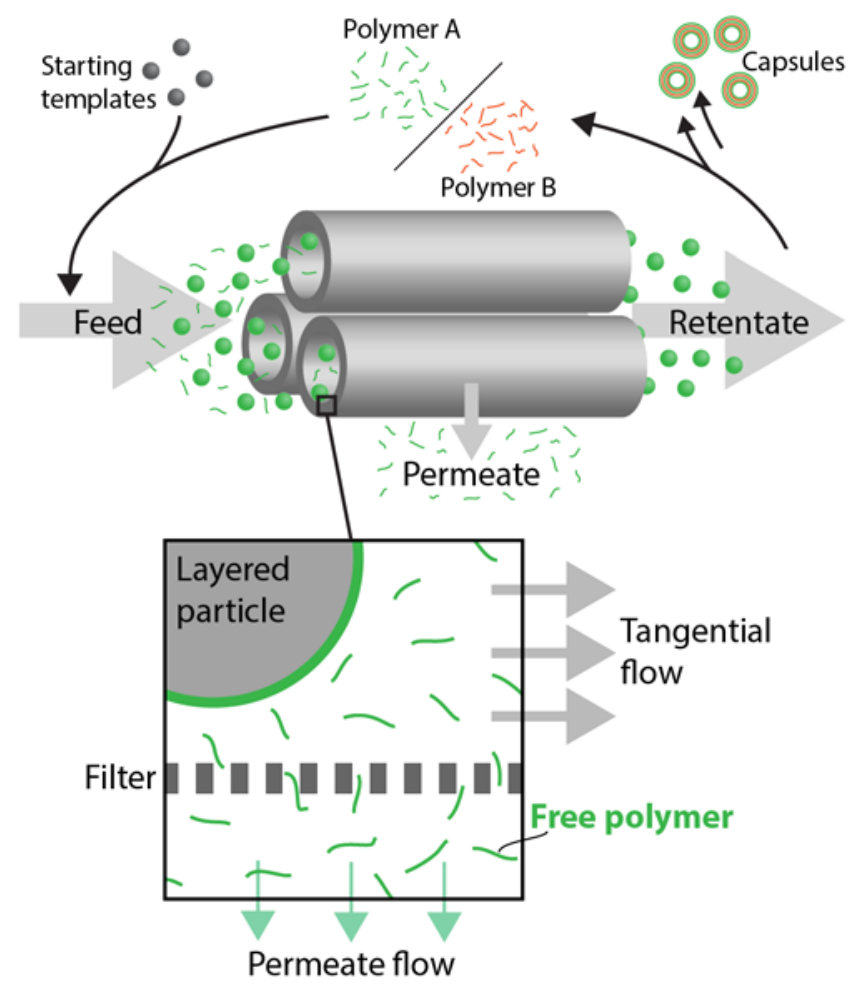


Figure 1. Hollow fiber tangential flow filtration (TFF) LbL assembly of capsules. Particulate templates are mixed with polymer A and, after incubation, purified using TFF. The layered and purified particles can then be mixed with the complementary polymer B to create a second layer. This process of TFF purification and LbL film deposition can then be repeated, yielding coreshell particles or capsules after core dissolution.

\section{EXPERIMENTAL SECTION}

Manual TFF LbL Assembly. Pre-washing of particles and incubation with polymer to deposit the first layer (either poly(allylamine hydrochloride) (PAH) or poly(diallyldimethylammonium chloride) (PDADMAC)) was performed as for standard centrifugation-based LbL assembly (15 min under agitation) (see Supporting Information for description of standard centrifugation-based LbL assembly) using the same amount of particles. After deposition was complete, the sample was transferred into a $15 \mathrm{~mL}$ conical centrifuge tube, diluted to $\sim 15 \mathrm{~mL}$ with ultrapure water, and aspirated into a syringe that was then connected to the TFF filter module. One syringe was also connected to the retentate line and a third syringe was connected to the permeate line (Figure S1 in Supporting Information). TFF was then performed by pushing the sample back and forth between the feed and retentate syringes (Video S1) until less than $\sim 2 \mathrm{~mL}$ sample volume remained. The permeate syringe was disconnected, the permeate discarded, and $25 \mathrm{~mL}$ ultrapure water was then added by back-flush through the permeate line. The washing procedure was then repeated two more times before the sample was recovered in a volume of $\sim 2-3 \mathrm{~mL}$, transferred to a $15 \mathrm{~mL}$ conical centrifuge tube, mixed with equal amount of poly(styrene sulfonate) (PSS) solution $\left(1 \mathrm{mg} \mathrm{mL}^{-1}\right.$, giving a final polymer concentration of $0.5 \mathrm{mg} \mathrm{mL}^{-1}$ ) and then incubated under constant agitation through rotation for 
15 min. The procedure was then repeated until four bilayers had been deposited. TFF filter modules with different pore sizes were used for the different particle sizes used in this study (Table S1).

Flow-based TFF LbL. Pumps, valves, tanks and tubing were used to replace the manual handling steps in manual TFF LbL (Figures S2 and S3). Layering was done in the system using continuous flow for $15 \mathrm{~min}$ in a layering loop instead of offline in a rotating conical centrifuge tube.

Characterization. Images were acquired using transmission electron microscopy (Philips CM120 BioTWIN), atomic force microscopy (tapping mode in air; NanoWizard II AFM, JPK), deconvolution fluorescence microscopy (DeltaVision, Applied Precision), and DIC and fluorescence microscopy (Olympus IX71). Particle concentration and fluorescence intensity of particles were measured on a flow cytometer (Apogee Micro Flow Cytometer). Zeta potential measurements were performed on a Zetasizer Nano ZS (Malvern) using a clear zeta cell for a minimum of 12 runs.

Statistics. The zeta potential measurements graphs provide the mean value (data points) and standard deviation (error bars) of the peaks, as provided by the instrument software (Zetasizer software, Malvern). Flow cytometry fluorescence intensity experiments were performed in triplicates and graphs provide the mean value (data points) and standard deviation (error bars) of the median fluorescence intensity as determined using a flow cytometry analysis software (FlowJo) (Figure S4). Particle counting was performed in triplicates and graphs provide the mean value (data point) and standard deviation (error bars) of the particle counts as determined by the concentration reported by the instrument software (Apogee Histogram) times the dilution factor and the sample volume. 
Additional experimental details are available in the Supporting Information.

\section{RESULTS AND DISCUSSION}

As a first example, silica particles (2.39 $\mu \mathrm{m}$ in diameter) were layered through standard incubation (in a rotating tube) with polymer and then washed using a TFF filter module (0.2 $\mu \mathrm{m}$ pore size) using a manual set-up where syringes are used to move the particle suspension through the filter, while collecting the permeate in a third syringe (Figure S1 and Video S1). The polymers used were the well-established model LbL polyelectrolyte pairs of PAH / PSS or PDADMAC / PSS. Zeta potential analysis showed charge reversal in each layering step, as expected when layering with oppositely charged polymers (Figure 2a). Fluorescence microscopy imaging of particles layered with fluorescein isothiocyanate (FITC)-labeled PAH indicated low background levels of free polymer after TFF washing (Figure 2b). Quantification of remaining polymer in the system further demonstrated successful washing and removal of free polymer (Figure S5) with the free polymer signal decreasing to background levels after washing with ten wash volumes (as suggested by the guidelines from the manufacturer of the TFF filter modules). Successful washing was also indicated by the absence of any visible formation of precipitates when introducing the oppositely charged polymer into the system (the used polymer pairs can easily aggregate and form complexes if significant amounts of free polymers are available in solution at the same time). Atomic force microscopy (AFM) imaging demonstrated that air-dried capsules folded and formed creases (Figure 2c), which is typical for polyelectrolyte capsules. ${ }^{13}$ Wall thicknesses of $\sim 12 \mathrm{~nm}$ were obtained from these AFM images, corresponding to $\sim 1.5 \mathrm{~nm}$ thickness per layer (Figure 2d). The root-mean-square roughness of the film was $\sim 2-3 \mathrm{~nm}$. The 
values for both layer thickness and roughness are similar to values reported for capsules of the same polymer pair prepared using similar conditions in centrifugation-based assembly. ${ }^{9}$

After confirming that microparticles can be layered, the layering of sub-micrometer-sized particles was investigated. Silica particles (519 $\mathrm{nm}$ in diameter) were layered with four bilayers of PAH-FITC/PSS (Figure 3). The fluorescence intensity of the particles was measured after layering, and the observed increase in fluorescence intensity indicates that PAH-FITC was successfully deposited on the particle surface. Following core-removal, deconvolution fluorescence microscopy demonstrated that capsules were formed. The coating of two other sizes of sub-micrometer particles (889 $\mathrm{nm}$ and $177 \mathrm{~nm}$ in diameter) was also investigated. After layering, transmission electron microscopy (TEM) was used to investigate the film structures of the core-shell particles and the capsules (Figure 4). The difference in contrast between the surface and the core for the core-shell particles indicates that the particles were successfully coated with a thin polymer film on the order of nanometers in thickness, which corresponds well to the AFM measurements (Figure 2c,d). Successful formation of capsules after core removal further demonstrates successful layering (Figure 4). 
a
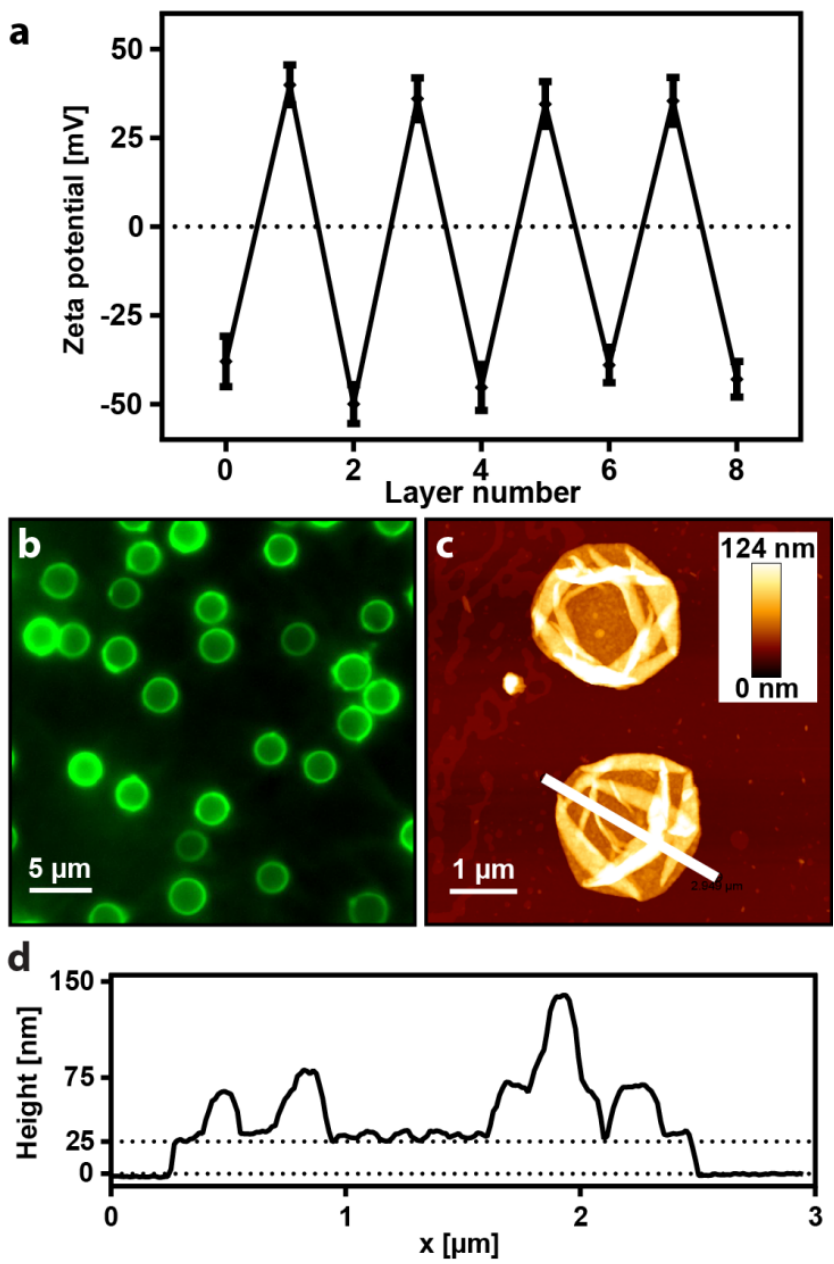

Figure 2. Microparticles can be layered using LbL assembly and TFF washing. (a) Zeta potential as a function of the number of layers. Layer 0 represents the bare silica particles (2.39 $\mu \mathrm{m}$ in diameter) before layering. Odd layer numbers are PDADMAC and even layer numbers are PSS. (b) Fluorescence microscopy image of $\mathrm{SiO}_{2}$ particles coated with (PAH-FITC/PSS) ${ }_{4}$ multilayers in solution. (c-d) AFM image and corresponding cross-section of air-dried $(\mathrm{PDADMAC} / \mathrm{PSS})_{4}$ capsules. Position of height profile in (d) indicated with white line in (c). Dotted line at $25 \mathrm{~nm}$ in (d) indicates approximate double wall thickness. 


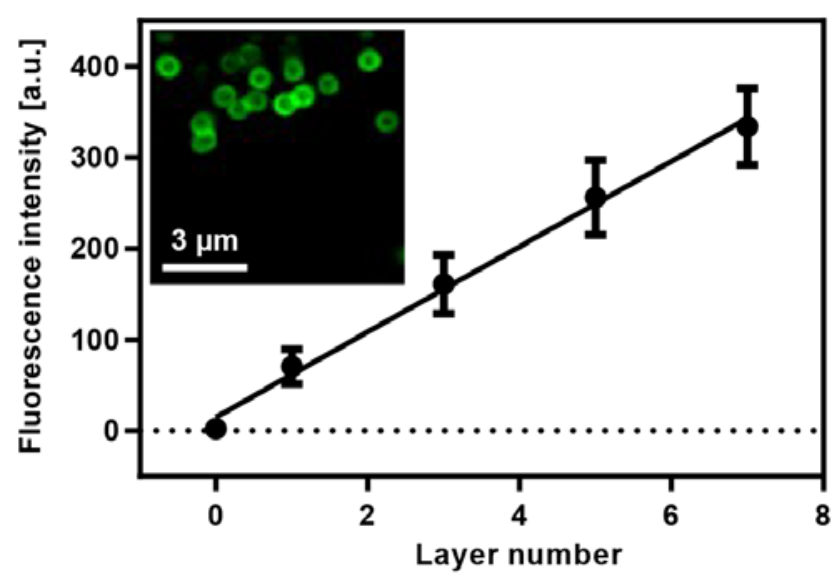

Figure 3. Sub-micrometer particles can be layered using LbL assembly and TFF washing. Increase in fluorescence intensity as a function of layer number measured using flow cytometry. Layer 0 represents bare particles before layering. Odd layer numbers corresponds to PAH-FITC. (Inset) Deconvolution microscopy image of four bilayer PAH-FITC/PSS capsules obtained from silica templates $(519 \mathrm{~nm})$.

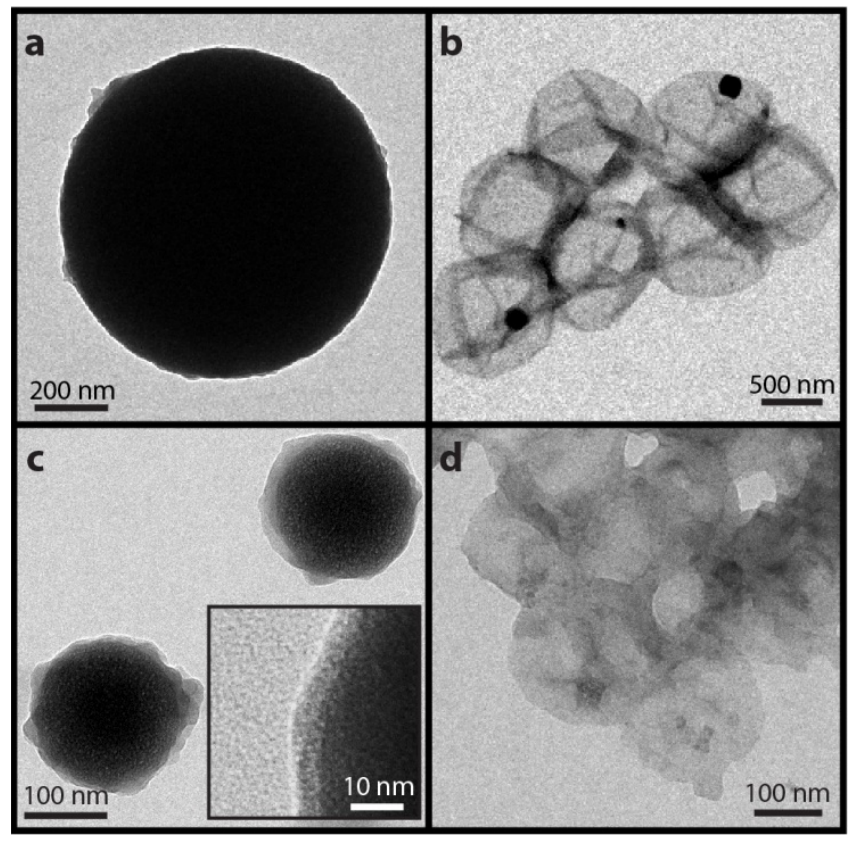

Figure 4. TEM of sub-micrometer core-shell particles and capsules prepared using LbL assembly and TFF washing. (PAH/PSS) ${ }_{4}$-coated silica templates and resulting capsules using 
$889 \mathrm{~nm}$ templates (a, coated template; b, capsules) and $177 \mathrm{~nm}$ templates (c, coated templates; d, capsules). (Inset) in (c) show higher magnification of surface of a (PAH/PSS) 4 -coated silica template $(177 \mathrm{~nm})$.

After confirming that TFF can be applied for the assembly of sub-micrometer particles and capsules using the manual TFF set-up, we designed a flow-based system to investigate the possibility of automation. The system was designed using two fluidic loops, one used during incubation with the polymer for layer deposition and one used during washing with the TFF filter (Figure 5a). Zeta potential analysis and fluorescence microscopy measurements demonstrated that capsules could be successfully assembled (Figure 5b,c).

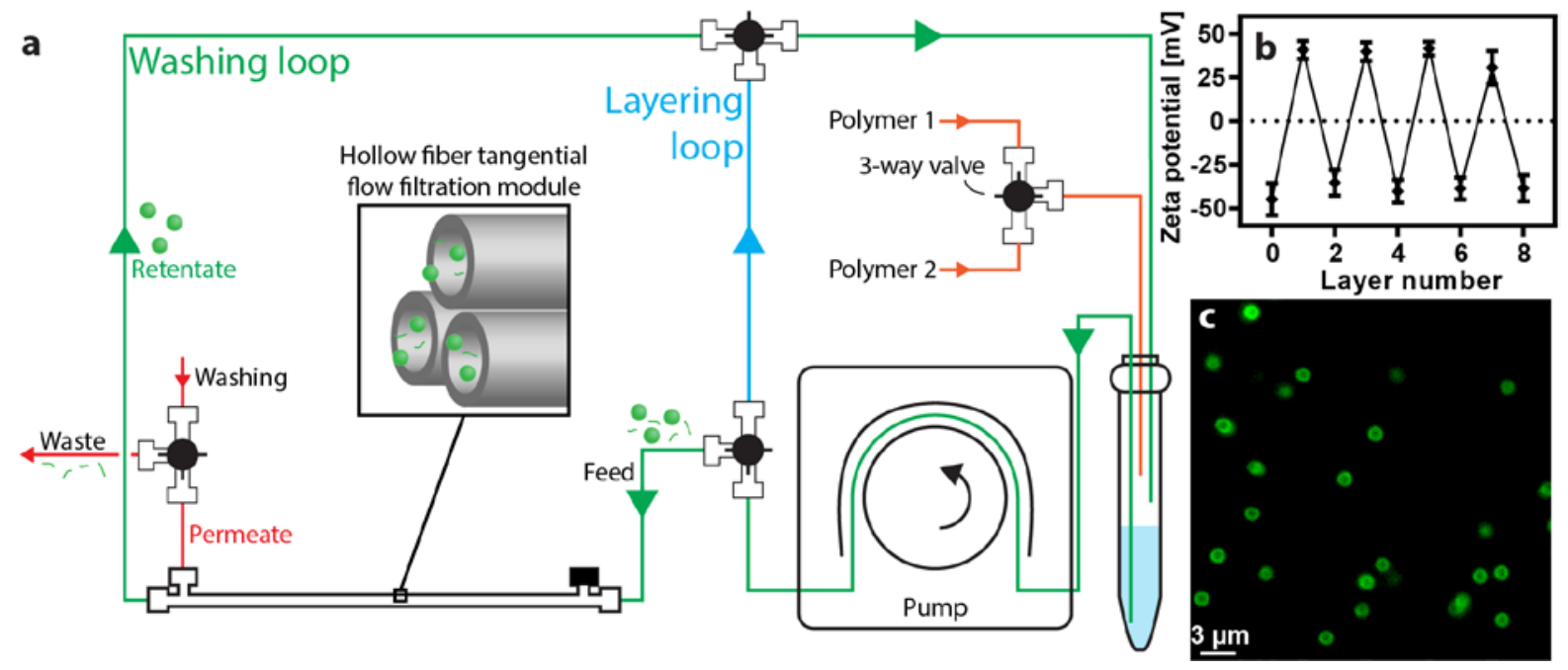

Figure 5. Flow-based TFF LbL assembly. (a) The system consists of a peristaltic pump and a reservoir with the particle suspension into which the polymers are added. A layering loop (blue) is used during incubation to achieve continuous flow in the system. A washing loop (green) onto which the TFF filter module is connected is used during the washing step. Back flush through the permeate line is used to add washing buffer sequentially during the washing. Additional 
information is available in Figures S2 and S3. (b) Zeta potential analysis of particle templates after layering with PAH-FITC (even numbers) and PSS (odd numbers). Layer 0 represents bare particles before layering. (c) (PAH-FITC/PSS) ${ }_{4}$ capsules obtained from silica templates (889 $\mathrm{nm})$.

Next, we investigated the possibility of centrifugation-free assembly of drug-loaded capsules. Silica templates are typically dissolved using hydrofluoric acid (HF) treatment, and the TFF filters used only have limited compatibility with HF (according to manufacturer's chemical compatibility chart). To circumvent this and to enable fully centrifugation-free assembly, we used porous calcium carbonate templates that have both high loading capacity and can easily be dissolved in mild conditions (e.g., using acetate buffer or ethylenediaminetetraacetic acid (EDTA)) ${ }^{40-43}$ compatible with the TFF filters (Figure 6a). The porous calcium carbonate templates ( $\sim 5 \mathrm{~m}$ in diameter) were loaded through incubation with doxorubicin (DOX), a drug used in cancer chemotherapy, and then added into the TFF LbL system. The calcium carbonate templates themselves are not highly charged, but after a bilayer had been deposited the difference in zeta potential for each layer increased, and the particles become significantly positively or negatively charged after each layering step, as expected when using oppositely charged polyelectrolytes for LbL assembly (Figure 6b). After the deposition of four bilayers, the core was dissolved by washing with EDTA solution and capsules were obtained (Figure 6c). Differential interference contrast (DIC) and fluorescence microscopy demonstrated that capsules containing both PAH-FITC and DOX had been successfully assembled.

Finally, we investigated the yield of the TFF LbL assembly system and compared it to the yield achieved during standard centrifugation-based LbL. Silica particles (889 nm in diameter) 
were layered with four bilayers of PAH/PSS with three centrifugation-based washing steps in each. Alternatively, particles from the same batch were layered with four bilayers of PAH/PSS (same batch as used for centrifugation-based LbL) using the TFF LbL system. The number of particles remaining after each layer was determined using flow cytometry (Figure 7).
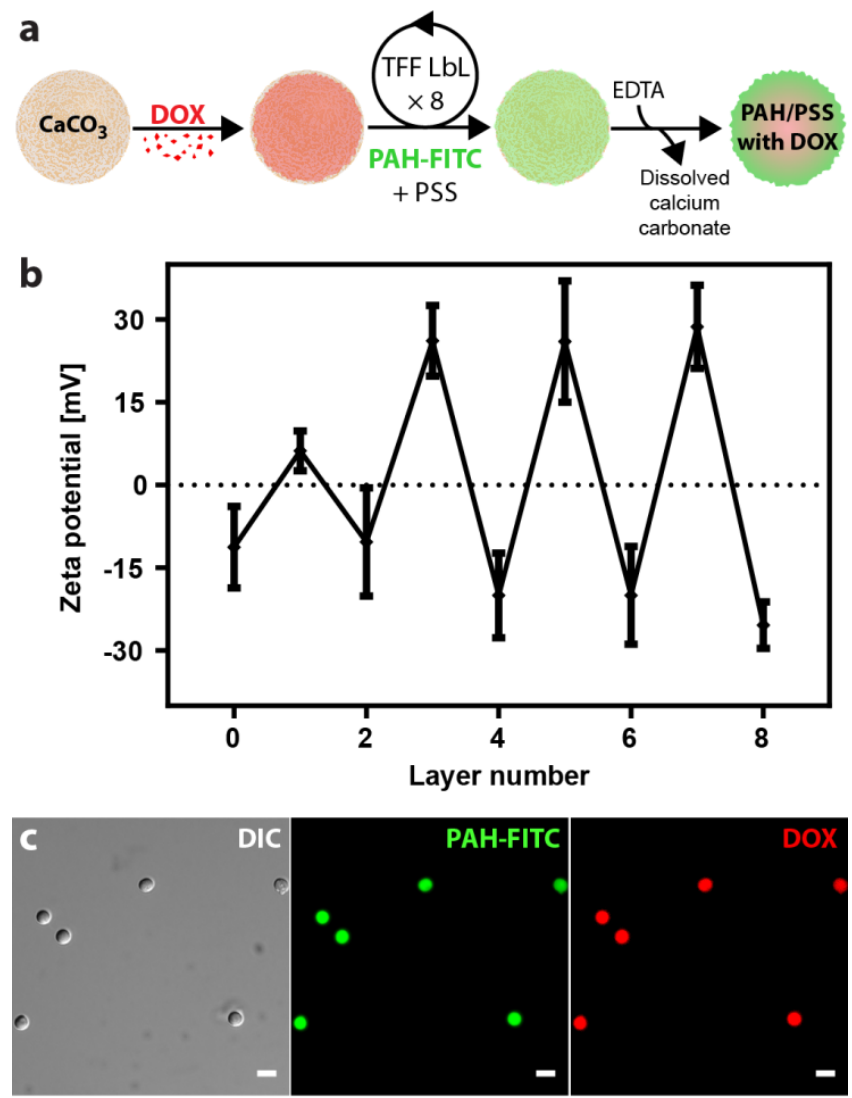

Figure 6. Centrifugation-free production of drug-loaded capsules. (a) Overview of the drugloading and layering process. DOX was loaded into the calcium carbonate templates that were then loaded into the flow-based TFF LbL system. After four bilayers of PAH-FITC/PSS had been deposited, EDTA was added to dissolve the core particles, resulting in DOX-loaded $(\mathrm{PAH} / \mathrm{PSS})_{4}$ capsules. (b) Zeta potential as a function of the number of layers. Layer 0 represents calcium carbonate particles loaded with DOX prior to layering. Odd layer numbers are PAH-FITC and even layer numbers are PSS. (c) DIC and fluorescence microscopy images obtained using green (PAH-FITC) and red (DOX) filter cubes. Scale bars are $5 \mu \mathrm{m}$. 


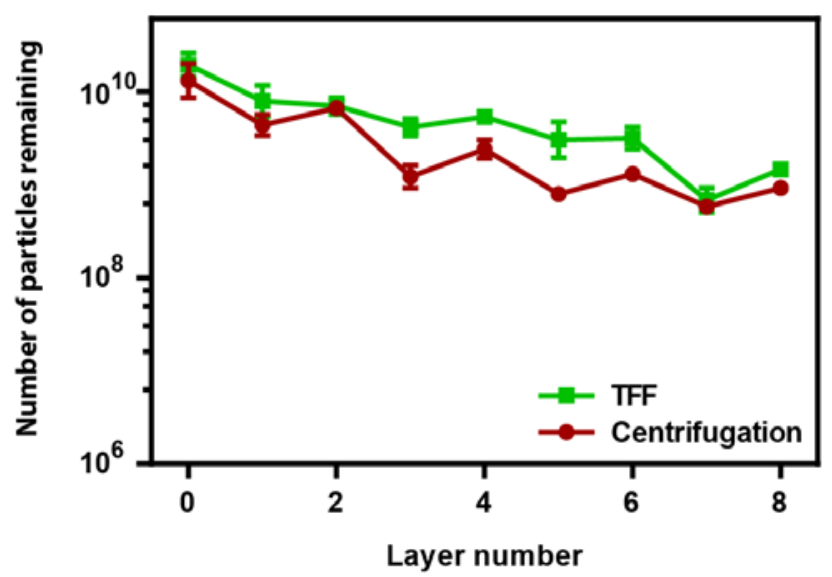

Figure 7. Yield of TFF LbL assembly. The number of particles remaining as a function of layer number with centrifugation-based LbL (red circles) or TFF LbL (green squares) as determined by flow cytometry. Layer 0 represents bare particles before layering. Odd layer numbers correspond to PAH and even layer numbers to PSS. Error bars for some data points are too small to be visible as they are within the size of the data points.

Centrifugation-based LbL assembly and TFF LbL assembly showed similar behavior in particle loss after each layer deposition and the total particle count decreased approximately an order of magnitude for both systems (total particle count decreased from $\sim 10^{10}$ to $\sim 10^{9}$ ). The multiple washing steps involved in centrifugation-based assembly (three washing steps per layer multiplied by eight layers equals 24 washing steps) means that even if one recovers $90-95 \%$ of particles per washing step the total loss still adds up to around $80-90 \%\left(0.9^{24} \approx 0.1\right)$, a fact wellknown for LbL systems and multi-step assembly. ${ }^{9,22}$ An advantage of many closed-loop systems is that they can aid in minimizing losses. To investigate the reason for particle loss during TFF LbL assembly, we disassembled the filter modules and imaged them using scanning electron 
microscopy (SEM; Figures S6-S9). The images show a highly porous network of the filters and that this network consists of both micrometer- and nanometer-sized structures. When comparing a filter before and after use (Figures S8 and S9), it is clear that particles coat the inner surfaces of the filter and that this is a significant contribution to the loss. The inherent propensity of widely used LbL materials, such as the polyelectrolytes used in this study (PAH and PSS), to coat most surfaces is what makes the technique so useful, but is also what can cause losses when particles and polymers adsorb onto other surfaces, such as filter membranes (a property that has been exploited to make functional membranes ${ }^{44-47}$ ). In this study, we re-used the same filters for several batches of particles with intermediate washing using just ultrapure water, although the filters are also compatible with ethanol or sodium hydroxide. We have discovered that simple back-flush through the permeate line of the filter aids in particle recovery, which is why the presented system includes this in the design. Pre-filtering of polymer solutions (prior to addition to the particle templates) to remove dust and contaminating particulate matter can also aid in increasing filter module performance. The TFF filter modules used in the current study are commercially available and use hydrophilically modified (proprietary) polyethersulfone membranes. Using other membrane chemistries and/or pre-treating the membranes may also increase performance. ${ }^{48-51}$

A standard incubation time of 15 min per adsorption step was used for both the flowbased system and for manual centrifugation-based assembly, so even though washing time was decreased (from typically 15 min for centrifugation-based assembly to $<5$ min for flow-based assembly) the total time per layer is still limited by the adsorption incubation time. However, for the flow-based system this can potentially be circumvented, as highly controlled flow conditions can allow for quicker layering (e.g., a 1 min deposition step was used in a fluidized bed 
system $^{29}$ ). Furthermore, for centrifugation-based assembly all steps were performed manually, while for the flow-based system only simple operations such as switching of valves and the addition of washing buffer were performed by the user. These operations have the potential to be fully automated (as routinely undertaken in industry), thus creating an integrated and automated closed-loop flow-based LbL system (Figure S3).

\section{CONCLUSIONS}

This study demonstrates the use of TFF for flow-based LbL assembly on particulate templates to generate multilayered particles and capsules. A wide size range of particles, from micrometer to sub-micrometer in diameter, were layered with polymer thin films. The film properties of the assembled capsules, as assessed using AFM, TEM, DIC and fluorescence microscopy imaging as well as AFM thickness and roughness measurements, are similar to those prepared by conventional centrifugation-based LbL assembly. Through judicious choice of layering material, template and pore size of the TFF filter module, TFF LbL assembly has the potential to be used for even smaller templates than the $177 \mathrm{~nm}$ reported here, which can be of interest for certain applications. Further, by using calcium carbonate templates that can be easily dissolved in conditions compatible with the TFF LbL assembly system, we demonstrated centrifugation-free assembly of drug-loaded capsules. The possibility to perform all LbL assembly steps, including core dissolution, inside a flow-based closed system, combined with the potential for scaled-up, fully automated and controlled multilayer particle and capsules production, makes TFF LbL assembly valuable for research environments, but also possibly useful for translating research into applications. 


\section{ASSOCIATED CONTENT}

Supporting Information. Additional experimental details, figures, schematics and a video. This material is available free of charge via the Internet at http://pubs.acs.org.

\section{AUTHOR INFORMATION}

\section{Corresponding Author}

*E-mail: fcaruso@unimelb.edu.au.

\section{Notes}

The authors declare no competing financial interest. ${ }^{\dagger}$ Present address: CSIRO Manufacturing Flagship, CSIRO Private Bag 10, Clayton South, Victoria 3169, Australia

\section{ACKNOWLEDGMENT}

This research was conducted and funded by the Australian Research Council Centre of Excellence in Convergent Bio-Nano Science and Technology (project number CE140100036). This work was also supported by the Australian Research Council (ARC) under the Australian Laureate Fellowship (F.C., FL120100030) and the Australian Government through an Australian Postgraduate Award (M.B.). This work was performed in part at the Materials Characterisation and Fabrication Platform (MCFP) at the University of Melbourne and the Victorian Node of the Australian National Fabrication Facility (ANFF). 


\section{REFERENCES}

(1) Decher, G. Fuzzy Nanoassemblies: Toward Layered Polymeric Multicomposites. Science 1997, 277, 1232-1237.

(2) Ariga, K.; Yamauchi, Y.; Rydzek, G.; Ji, Q.; Yonamine, Y.; Wu, K. C.-W.; Hill, J. P. Layer-by-Layer Nanoarchitectonics: Invention, Innovation, and Evolution. Chem. Lett. 2014, 43, 36-68.

(3) De Koker, S.; Hoogenboom, R.; De Geest, B. G. Polymeric Multilayer Capsules for Drug Delivery. Chem. Soc. Rev. 2012, 41, 2867-2884.

(4) Yan, Y.; Björnmalm, M.; Caruso, F. Assembly of Layer-by-Layer Particles and Their Interactions with Biological Systems. Chem. Mater. 2014, 26, 452-460.

(5) Peyratout, C. S.; Dähne, L. Tailor-Made Polyelectrolyte Microcapsules: From Multilayers to Smart Containers. Angew. Chem. Int. Ed. 2004, 43, 3762-3783.

(6) Yan, Y.; Björnmalm, M.; Caruso, F. Particle Carriers for Combating Multidrug-Resistant Cancer. ACS Nano 2013, 7, 9512-9517.

(7) Tong, W.; Song, X.; Gao, C. Layer-by-Layer Assembly of Microcapsules and Their Biomedical Applications. Chem. Soc. Rev. 2012, 41, 6103-6124.

(8) Hammond, P. T. Building Biomedical Materials Layer-by-Layer. Mater. Today 2012, 15, 196-206.

(9) Richardson, J. J.; Björnmalm, M.; Caruso, F. Technology-Driven Layer-by-Layer Assembly of Nanofilms. Science 2015, 348, aaa2491.

(10) Borges, J.; Mano, J. F. Molecular Interactions Driving the Layer-by-Layer Assembly of Multilayers. Chem. Rev. 2014, 114, 8883-8942.

(11) Ariga, K.; Hill, J. P.; Ji, Q. Layer-by-Layer Assembly as a Versatile Bottom-up Nanofabrication Technique for Exploratory Research and Realistic Application. Phys. Chem. Chem. Phys. 2007, 9, 2319-2340.

(12) Caruso, F.; Caruso, R. A.; Möhwald, H. Nanoengineering of Inorganic and Hybrid Hollow Spheres by Colloidal Templating. Science 1998, 282, 1111-1114.

(13) Donath, E.; Sukhorukov, G. B.; Caruso, F.; Davis, S. A.; Möhwald, H. Novel Hollow Polymer Shells by Colloid-Templated Assembly of Polyelectrolytes. Angew. Chem. Int. Ed. 1998, 37, 2201-2205. 
(14) Sukhorukov, G. B.; Donath, E.; Lichtenfeld, H.; Knippel, E.; Knippel, M.; Budde, A.; Möhwald, H. Layer-by-Layer Self Assembly of Polyelectrolytes on Colloidal Particles. Colloids Surf., A. 1998, 137, 253-266.

(15) Richardson, J. J.; Ejima, H.; Lörcher, S. L.; Liang, K.; Senn, P.; Cui, J.; Caruso, F. Preparation of Nano- and Microcapsules by Electrophoretic Polymer Assembly. Angew. Chem. Int. Ed. 2013, 52, 6455-6458.

(16) Morton, S. W.; Herlihy, K. P.; Shopsowitz, K. E.; Deng, Z. J.; Chu, K. S.; Bowerman, C. J.; Desimone, J. M.; Hammond, P. T. Scalable Manufacture of Built-to-Order Nanomedicine: Spray-Assisted Layer-by-Layer Functionalization of PRINT Nanoparticles. Adv. Mater. 2013, 25, 4707-4713.

(17) Richardson, J. J.; Liang, K.; Kempe, K.; Ejima, H.; Cui, J.; Caruso, F. Immersive Polymer Assembly on Immobilized Particles for Automated Capsule Preparation. Adv. Mater. 2013, 25, 6874-6878.

(18) Richardson, J. J.; Björnmalm, M.; Gunawan, S. T.; Guo, J.; Liang, K.; Tardy, B.; Sekiguchi, S.; Noi, K. F.; Cui, J.; Ejima, H.; Caruso, F. Convective Polymer Assembly for the Deposition of Nanostructures and Polymer Thin Films on Immobilized Particles. Nanoscale 2014, 6, 13416-13420.

(19) Qi, A.; Chan, P.; Ho, J.; Rajapaksa, A.; Friend, J.; Yeo, L. Template-Free Synthesis and Encapsulation Technique for Layer-by-Layer Polymer Nanocarrier Fabrication. ACS Nano 2011, 5, 9583-9591.

(20) Hong, X.; Li, J.; Wang, M.; Xu, J.; Guo, W.; Li, J.; Bai, Y.; Li, T. Fabrication of Magnetic Luminescent Nanocomposites by a Layer-by-Layer Self-Assembly Approach. Chem. Mater. 2004, 16, 4022-4027.

(21) Grigoriev, D. O.; Bukreeva, T.; Möhwald, H.; Shchukin, D. G. New Method for Fabrication of Loaded Micro- and Nanocontainers: Emulsion Encapsulation by Polyelectrolyte Layer-by-Layer Deposition on the Liquid Core. Langmuir 2008, 24, 9991004.

(22) Voigt, A.; Lichtenfeld, H.; Sukhorukov, G. B.; Zastrow, H.; Donath, E.; Bäumler, H.; Möhwald, H. Membrane Filtration for Microencapsulation and Microcapsules Fabrication by Layer-by-Layer Polyelectrolyte Adsorption. Ind. Eng. Chem. Res. 1999, 38, 40374043.

(23) Hirsjärvi, S.; Peltonen, L.; Hirvonen, J. Layer-by-Layer Polyelectrolyte Coating of Low Molecular Weight Poly(lactic Acid) Nanoparticles. Colloids Surf., B 2006, 49, 93-99.

(24) Hirsjärvi, S.; Qiao, Y.; Royere, A.; Bibette, J.; Benoit, J.-P. Layer-by-Layer Surface Modification of Lipid Nanocapsules. Eur. J. Pharm. Biopharm. 2010, 76, 200-207. 
(25) Priest, C.; Quinn, A.; Postma, A.; Zelikin, A. N.; Ralston, J.; Caruso, F. Microfluidic Polymer Multilayer Adsorption on Liquid Crystal Droplets for Microcapsule Synthesis. Lab Chip 2008, 8, 2182-2187.

(26) Kantak, C.; Beyer, S.; Yobas, L.; Bansal, T.; Trau, D. A “Microfluidic Pinball” for onChip Generation of Layer-by-Layer Polyelectrolyte Microcapsules. Lab Chip 2011, 11, 1030-1035.

(27) Björnmalm, M.; Yan, Y.; Caruso, F. Engineering and Evaluating Drug Delivery Particles in Microfluidic Devices. J. Control. Release 2014, 190, 139-149.

(28) Matosevic, S.; Paegel, B. M. Layer-by-Layer Cell Membrane Assembly. Nat. Chem. 2013, 5, 958-963.

(29) Richardson, J. J.; Teng, D.; Björnmalm, M.; Gunawan, S. T.; Guo, J.; Cui, J.; Franks, G. V; Caruso, F. Fluidized Bed Layer-by-Layer Microcapsule Formation. Langmuir 2014, 30, 10028-10034.

(30) Kaufman, G.; Boltyanskiy, R.; Nejati, S.; Thiam, A. R.; Loewenberg, M.; Dufresne, E. R.; Osuji, C. O. Single-Step Microfluidic Fabrication of Soft Monodisperse Polyelectrolyte Microcapsules by Interfacial Complexation. Lab Chip 2014, 14, 3494-3497.

(31) Parker, R. M.; Zhang, J.; Zheng, Y.; Coulston, R. J.; Smith, C. A.; Salmon, A. R.; Yu, Z.; Scherman, O. A.; Abell, C. Electrostatically Directed Self-Assembly of Ultrathin Supramolecular Polymer Microcapsules. Adv. Funct. Mater. 2015, 25, 4091-4100.

(32) Wang, Q.; Schlenoff, J. B. Single- and Multicompartment Hollow Polyelectrolyte Complex Microcapsules by One-Step Spraying. Adv. Mater. 2015, 27, 2077-2082.

(33) Merrifield, R. B. Automated Synthesis of Peptides. Science 1965, 150, 178-185.

(34) Caruthers, M. H. Gene Synthesis Machines: DNA Chemistry and Its Uses. Science 1985, 230, 281-285.

(35) Li, J.; Ballmer, S. G.; Gillis, E. P.; Fujii, S.; Schmidt, M. J.; Palazzolo, A. M. E.; Lehmann, J. W.; Morehouse, G. F.; Burke, M. D. Synthesis of Many Different Types of Organic Small Molecules Using One Automated Process. Science 2015, 347, 1221-1226.

(36) Service, R. F. The Synthesis Machine. Science 2015, 347, 1190-1193.

(37) Genovesi, C. S. Several Uses for Tangential-Flow Filtration in the Pharmaceutical Industry. PDA J. Pharm. Sci. Technol. 1983, 37, 81-86.

(38) Van Reis, R.; Leonard, L. C.; Hsu, C. C.; Builder, S. E. Industrial Scale Harvest of Proteins from Mammalian Cell Culture by Tangential Flow Filtration. Biotechnol. Bioeng. 1991, 38, 413-422. 
(39) Van Reis, R.; Gadam, S.; Frautschy, L. N.; Orlando, S.; Goodrich, E. M.; Saksena, S.; Kuriyel, R.; Simpson, C. M.; Pearl, S.; Zydney, A. L. High Performance Tangential Flow Filtration. Biotechnol. Bioeng. 1997, 56, 71-82.

(40) Sukhorukov, G. B.; Volodkin, D. V.; Günther, A. M.; Petrov, A. I.; Shenoy, D. B.; Möhwald, H. Porous Calcium Carbonate Microparticles as Templates for Encapsulation of Bioactive Compounds. J. Mater. Chem. 2004, 14, 2073-2081.

(41) Volodkin, D. V.; Larionova, N. I.; Sukhorukov, G. B. Protein Encapsulation via Porous CaCO3 Microparticles Templating. Biomacromolecules 2004, 5, 1962-1972.

(42) Wang, C.; He, C.; Tong, Z.; Liu, X.; Ren, B.; Zeng, F. Combination of Adsorption by Porous CaCO3 Microparticles and Encapsulation by Polyelectrolyte Multilayer Films for Sustained Drug Delivery. Int. J. Pharm. 2006, 308, 160-167.

(43) Richardson, J. J.; Maina, J. W.; Ejima, H.; Hu, M.; Guo, J.; Choy, M. Y.; Gunawan, S. T.; Lybaert, L.; Hagemeyer, C. E.; Geest, B. G. De; Caruso, F. Versatile Loading of Diverse Cargo into Functional Polymer Capsules. Adv. Sci. 2015, 2, 1400007.

(44) Bruening, M. L.; Sullivan, D. M. Enhancing the Ion-Transport Selectivity of Multilayer Polyelectrolyte Membranes. Chem. Eur. J. 2002, 8, 3832-3837.

(45) Jin, W.; Toutianoush, A.; Tieke, B. Use of Polyelectrolyte Layer-by-Layer Assemblies as Nanofiltration and Reverse Osmosis Membranes. Langmuir 2003, 19, 2550-2553.

(46) Krogman, K. C.; Lowery, J. L.; Zacharia, N. S.; Rutledge, G. C.; Hammond, P. T. Spraying Asymmetry into Functional Membranes Layer-by-Layer. Nat. Mater. 2009, 8, 512-518.

(47) Bruening, M. L. Creating Functional Membranes through Polyelectrolyte Adsorption. In Multilayer Thin Films: Sequential Assembly of Nanocomposite Materials; Decher, G., Schlenoff, J. B., Eds.; Wiley-VCH: Weinheim, Germany, 2012; Vol. 2, pp 907-924.

(48) Trägårdh, G. Membrane Cleaning. Desalination 1989, 71, 325-335.

(49) Shi, X.; Tal, G.; Hankins, N. P.; Gitis, V. Fouling and Cleaning of Ultrafiltration Membranes: A Review. J. Water Process Eng. 2014, 1, 121-138.

(50) Schaefer, A. I.; Fane, A. G.; Waite, T. D. Nanofiltration: Principles and Applications, 1st ed.; Elsevier B.V., Amsterdam, Netherlands, 2004.

(51) Sutherland, K. Filters and Filtration Handbook, 5th ed.; Elsevier B.V., Amsterdam, Netherlands, 2008. 
TABLE OF CONTENTS GRAPHIC

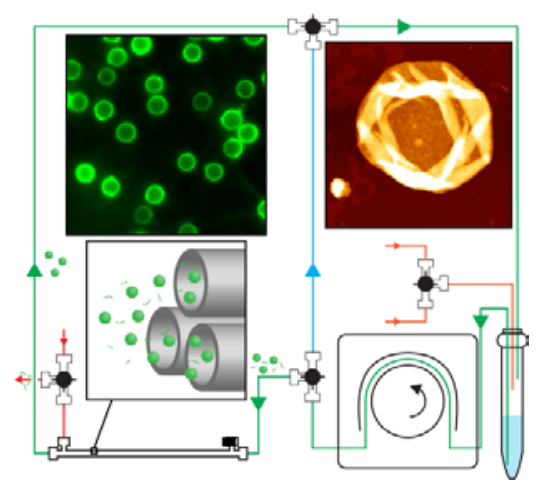




\section{University Library}

\section{- M M I E E R VA A gateway to Melbourne's research publications}

Minerva Access is the Institutional Repository of The University of Melbourne

Author/s:

Bjoernmalm, M;Roozmand, A;Noi, KF;Guo, J;Cui, J;Richardson, JJ;Caruso, F

Title:

Flow-Based Assembly of Layer-by-Layer Capsules through Tangential Flow Filtration

Date:

2015-08-25

Citation:

Bjoernmalm, M., Roozmand, A., Noi, K. F., Guo, J., Cui, J., Richardson, J. J. \& Caruso, F. (2015). Flow-Based Assembly of Layer-by-Layer Capsules through Tangential Flow Filtration. LANGMUIR, 31 (33), pp.9054-9060. https://doi.org/10.1021/acs.langmuir.5b02099.

Persistent Link:

http://hdl.handle.net/11343/90855 